Casos Clínicos

Arch. Esp. Urol., 59, 5 (533-535), 2006
Palabras clave: Dolor pélvico crónico. Incontinencia. Cistouretropexia.

\section{DOLOR PÉLVICO CRÓNICO COMO COMPLICACIÓN DE LA CISTOURETROPEXIA CON MALLA LIBRE DE TENSIÓN. PRESENTACIÓN DE UN CASO.}

Javier del Rosario, Rubén Ureña, Alejandro Manduley y Lilia Córdoba.

Servicio de Urología. Complejo Hospitalario Metropolitano de la Caja del Seguro Social de Panamá. Panamá.

Resumen.- OBJETIVO: presentar en la comunidad urológica un caso de interés de una complicación poco común de un procedimiento ya muy extendido como es la cistouretropexia, así como su diagnóstico preciso y resultados al tratamiento.

MÉTODO: Se revisó la historia clínica de la paciente así como el protocolo quirúrgico y se le dio seguimiento en la consulta externa por los 6 meses siguientes. Se presentan los hallazgos de manera descriptiva.

RESULTADOS: Se trata de una paciente que presenta un rechazo a tejido foráneo por una malla libre de tensión suburetral, quien cursa con dolor intenso en el postoperatorio mediato que lleva al retiro de la misma pese a que el resultado de continencia se había logrado.

CONCLUSIONES: El recazo a tejido foráneo es una complicación rara de las malas vaginales por lo cual es poco tomada en cuenta, hay que tener presente que es un diagnóstico de exclusión y que la posibilidad de retiro de la misma no en todos los casos conlleva reaparición de la incontinencia.
Alejandro Manduley C.

Apartado postal 89-9973.

Panamá. (Rep. de Panamá).

Alejandro_manduley@hotmail.com

Trabajo recibido: 8 de julio 2005
Summary.- OBJECTIVE: To report an interesting case of a rare complication of cystourethropexy, as well as its precise diagnosis and results after treatment.

METHODS: We reviewed the medical records of the patients as well as their operative report and she underwent outpatient follow-up for the following six months. We descriptively report the findings.

RESULTS: It is the case of a female patient presenting a foreign body rejection of a suburethral tension free vaginal tape (TVT). She complained of severe pain in the immediate postoperative period, which led to retrieval of the tape in spite of a good continence result.

CONCLUSIONS: Foreign body rejection is a rare complication of vaginal tapes; it is rarely taken into account but it should be present in any exclusion diagnosis because the possibility of tape retrieval is not always associated with recurrence of the incontinence.

Keywords: Pelvic pain. Incontinence. Cystourethropexy.

\section{CASO CLÍNICO}

Se trata de paciente femenina de 51 años con historia de salida involuntaria de orina al realizar esfuerzos, de aproximadamente 3 años de evolución, progresiva, sin urgencia ni síntomas irritativos. Sin prolapso vaginal anterior ni posterior. Sin antecedentes de cirugía pélvica.

La paciente fue programada de manera electiva para cistouretropexia con malla libre de tensión con mala de Prolene cortada de $10 \mathrm{~cm} \times 1 \mathrm{~cm}$, suturas de Prolene 2-0 y pasadores de IVS $\circledast$.

La cirugía se realizó sin complicaciones y con control cistoscópico, se retiró la sonda a las 24 horas y se egresó con adecuada calidad miccional, sin escape ni sensación de vaciado incompleto.

Acude a la consulta externa en la 3ra semana postoperatoria por presentar dolor en área suprapúbica e hipogastrio no irradiado, no asociado a la micción, de intensidad moderada a severa llegando a ser incapacitante en ocasiones.

Refiere buena calidad miccional y corrección de la incontinencia de esfuerzo, niega síntomas de urgencia. 


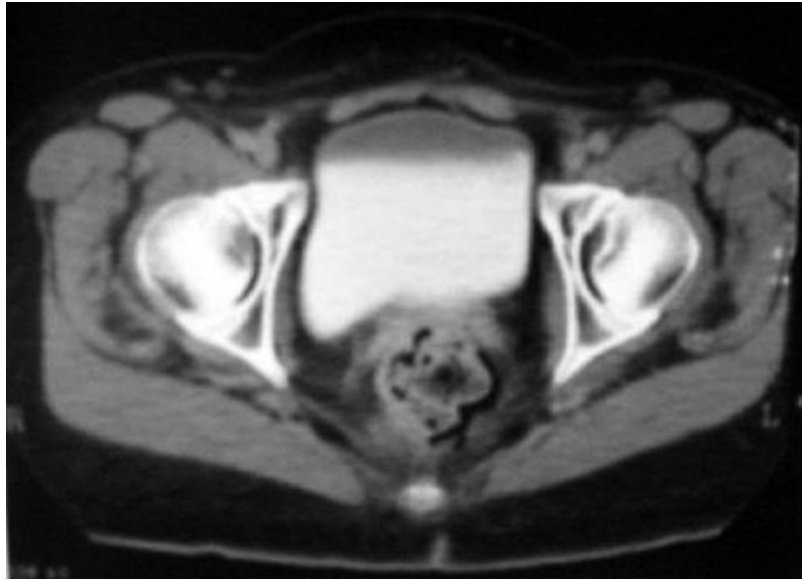

FIGURA 1. TAC de pelvis sin hallazgos anatómicos evidentes que explique el cuadro.

La paciente se encuentra en buen estado general, afebril, sin leucocitosis ni datos clínicos o laboratoriales de infección urinaria.

Con dolor intenso a la palpación superficial y profunda en el área suprapúbica y en el área parauretral bilateral al examen vaginal. El resto del examen físico resultó normal.

Se le programa cistoscopía en la cual no se evidencia estrechez ni erosión de la malla. Sin hilos ni malla transvesicales.

Se le realiza USG pélvico sin hallazgos patológicos, sin colecciones.

Radiografía de pelvis sin datos sugestivos de erosión del pubis o de osteítis.

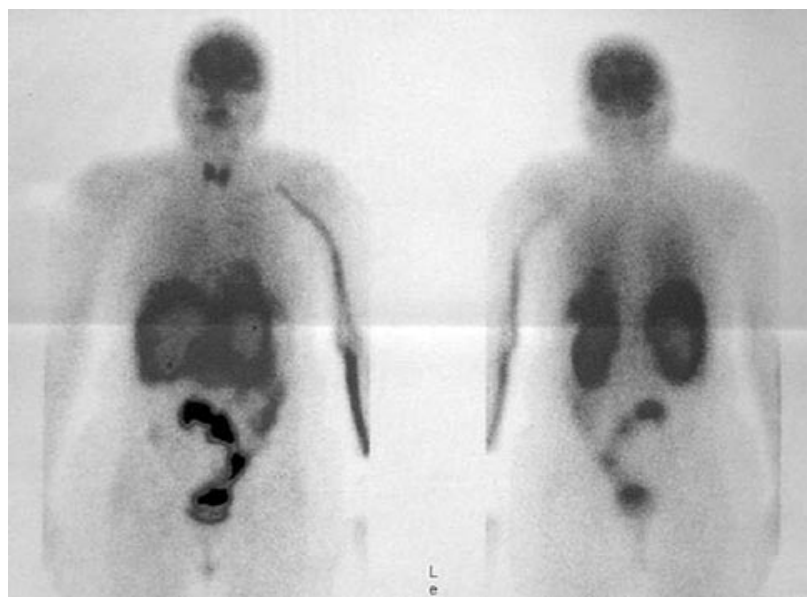

Se le realiza infiltración del área retropúbica con bupivacaína y corticoides en la semana 5 postoperatoria cursando con mejoría transitoria (24 horas) y reinstalación del cuadro.

Se consulta con medicina del dolor quienes recomiendan el manejo con opiáceos.

Se maneja con opiáceos orales con mejoría parcial del dolor y vuelta del mismo al suspender la medicación por lo cual se decide hospitalizar para completar estudios.

Se le realiza centelleo con leucocitos marcados en el que se informa un área de actividad inflamatoria localizada anterosuperiór a la vejiga y que se extiende hacia el hipogastrio lateralizado hacia la izquierda.

Tomografía pélvica dentro de límites normales.

Se decide levarla a salón de operaciones para la extracción de la malla el cual se realiza vía laparoscópica y transvaginal (combinado) logrando la extracción de la misma en su totalidad.

Cursa un postoperatorio satisfactorio y es dada de alta 3 días después con poco dolor el cual es tolerable con acetaminofén.

Se evalúa 2 meses después de la cirugía, refiere cursar con buena calidad miccional y sin dolor.

El dolor crónico criptogénico postoperatorio es una complicación muy poco común de los slings vaginales $(0,3 \%)$; encontrándose en la mayoría de los casos una causa subyacente como colección pélvica, erosión, cuerpo extraño intravesical, infección del sitio operatorio u osteítis pubis.

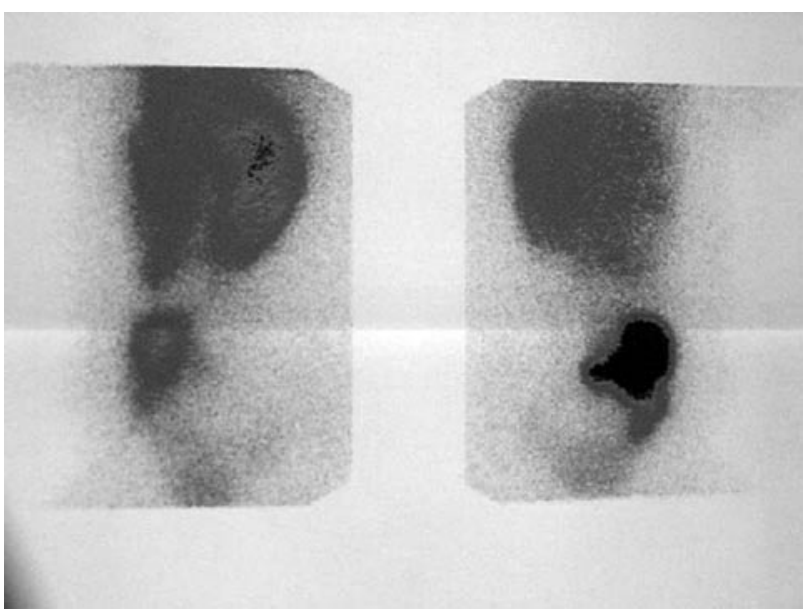

FIGURA 2. Centelleo con glóbulos blancos marcados en el que se impregna el área suprapúbica antevesical sugestivo de proceso inflamatorio persistente. 
El diagnóstco de rechazo a la malla es de exclusión dentro del cual quedaría el caso de esta paciente. En estos casos es mandatario la extracción total de la malla la cual se debe completar por un abordaje retropúbico perfectamente factible por laparoscopía.

El examen histopatológico de la paciente se reportó como inflamación inespecífica.

\section{BIBLIOGRAFIA y LECTURAS RECOMENDADAS (*lectura de interés $y^{* *}$ lectura fundamental)}

1. BHARGAVA, S.; CHAPPLE, C.R.: "Rising Awareness Of The Complications Of Synthetic Slings". Current Opinion In Urology, 14: 317, 2004.

2. LENG, W.W.; DAVIES, B.J.; TARIN, T.: "Delayed Treatment Of Bladder Outlet Obstruction After Sling Surgery: Association With Irreversible Bladder Dysfunction". Journal Of Urology. 172: 1379, 2004.

3. TSIA-SHU, L.: "Tension-Free Vaginal Tape Procedures In Women With Stress Urinary Incontinence With And Without Co-Existing Genital Prolapse. Current Opinion”. In Obstetrics \& Gynecology, 16: 399, 2004.

4. TSIVIAN, A.; MOGUTIN, B.; KESSLER, O. y cols.: "Ami Tension-Free Vaginal Tape Procedure For The Treatment Of Female Stress Urinary Incontinence: Long-Term Results". Journal Of Urology, 172: 998, 2004.

5. KIELB, S.J.; CLEMENS, J.Q.: "Endoscopic Excision Of Intravesical Tension-Free Vaginal Tape With Laparoscopic Instrument Assistance". Journal Of Urology, 172: 971, 2004.

6. EL-AZAB, A.S.; RACKLEY, R.R.; ABDELMALAK, J.B. y cols.: "Cystoscopic Resection Of Tension Free Vaginal Tape (Tvt) In The Bladder: A Novel Technique (Case Report)". Journal Of Pelvic Medicine \& Surgery, 10: 85, 2004.

7. KOBASHI, K.C.; GOVIER, F.E.: "Perioperative Complications: The First 140 Polypropylene Pubovaginal Slings". Journal Of Urology, 170: 1918, 2003.

8. RODRIGUEZ, L.V.; RAZ, S.: "Prospective Analysis Of Patients Treated With A Distal Urethral Polypropylene Sling For Symptoms Of Stress Urinary Incontinence: Surgical Outcome And Satisfaction Determined By Patient Driven Questionnaires". Journal Of Urology. 170: 857, 2003.

9. KOSBASHI, K.C.; GOVIER, F.E.: "Management Of Vaginal Erosion Of Polypropylene Mesh Slings". Journal Of Urology, 169: 2242, 2003.

10. KAPLAN, S.A.; TE, A.E.; YOUNG, G.P.H. y cols.: "Prospective Analysis Of 373 Consecutive Women With Stress Urinary Incontinence Treated With A Vaginal Wall Sling: The Columbia-Cornell University Experience". Journal Of Urology, 164: 1623, 2000.

11. PETROU, S.P.; BROWN, J.A.; BLAIVAS, J.G.: "Suprameatal transvaginal urethrolysis". Journal of urology, 161: 1268, 1999.
Casos Clínicos

Arch. Esp. Urol., 59, 5 (535-537), 2006

\section{SÍNDROME VURD CON DISPLASIA RENAL CONTRALATERAL AL REFLUJO EN EL ADULTO.}

José Manuel Otero García, Efraín Maldonado Alcaraz y Hugo Wingartz Plata.

Servicio de Urología. Hospital de Especialidades CMNSXXI Instituto Mexicano del Seguro Social. Méjico D.F. Méiico.

Resumen.- OBJETIVO: Presentar un caso de síndrome VURD (Valvas de uretra posterior, reflujo vesicoureteral y displasia renall, en el adulto.

MÉTODOS: Varón de 32 años con infección de vías urinarias de repetición, con estudios de gabinete y cistoscopia al cual se le diagnostica sindrome VURD.

RESULTADOS: Se resecan las valvas, al año de cirugía el paciente mantiene su función renal y adecuada micción sin necesidad de tratamiento dialítico.

CONCLUSIONES: El síndrome de VURD es un padecimiento congénito, que frecuentemente tiene un desenlace fatal en los primeros años de vida. La etiología no esta bien clara. Se ha planteado la relación causa efecto de displasia en relación a la obstrucción y reflujo; por otra parte, existe la "teoría de la yema", la cual sugiere que el reflujo vesico-ureteral (RVU) y la displasia renal son el resultado de una anormalidad en la yema ureteral y no necesariamente a la obstrucción y/o RVU per se.

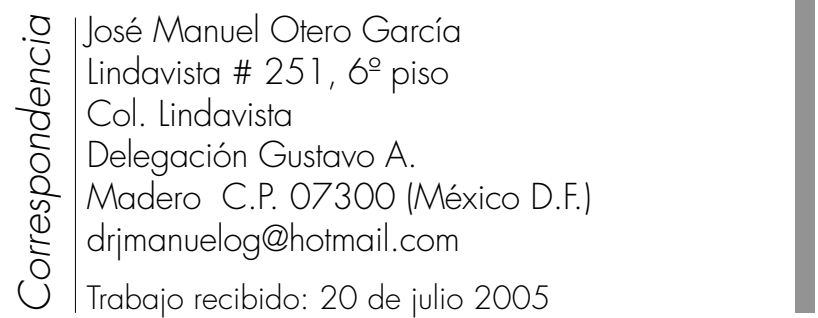

